\title{
Wide-Baseline Multi-View Video Segmentation For 3D Reconstruction
}

\author{
[Anonymous Submission]
}

\begin{abstract}
Obtaining a foreground silhouette across multiple views is one of the fundamental steps in 3D reconstruction. In this paper we present a novel video segmentation approach, to obtain a foreground silhouette, for scenes captured by a wide-baseline camera rig given a sparse manual interaction in a single view. The algorithm is based on trimap propagation, a framework used in video matting. Bayesian inference coupled with camera calibration information are used to spatio-temporally propagate high confidence trimap labels across the multi-view video to obtain coarse silhouettes which are later refined using a matting algorithm. Recent techniques have been developed for foreground segmentation, based on image matting, in multiple views but they are limited to narrow baseline with low foreground variation. The proposed wide-baseline silhouette propagation is robust to inter-view foreground appearance changes, shadows and similarity in foreground/background appearance. The approach has demonstrated good performance in silhouette estimation for views up to $180^{\circ}$ baseline (opposing views). The segmentation technique has been fully integrated in a multi-view reconstruction pipeline. The results obtained demonstrate the suitability of the technique for multi-view reconstruction with wide-baseline camera set-ups and natural background.
\end{abstract}

\section{Keywords}

Multi-view, wide-baseline, segmentation, silhouette propagation

\section{INTRODUCTION}

Obtaining a foreground silhouette across multiple views is a key step in $3 \mathrm{D}$ reconstruction of a captured scene. Automatic silhouette estimation is a difficult problem which is generally limited to narrow-baseline [6] or requires a large number of cameras [11]. A more practical camera setup requires considerably fewer cameras ( 8 in the proposed technique) to obtain a $3 \mathrm{D}$ reconstruction for free-viewpoint ren-

Permission to make digital or hard copies of all or part of this work for personal or classroom use is granted without fee provided that copies are not made or distributed for profit or commercial advantage and that copies bear this notice and the full citation on the first page. To copy otherwise, to republish, to post on servers or to redistribute to lists, requires prior specific permission and/or a fee.

Copyright 2007 ACM X-XXXXX-XX-X/XX/XX ...\$10.00. dering. This setup considerably increases the camera baseline and approaches like [6,7] can not be used as they use a hard constraint of invariant foreground appearance across multiple views. Due to the presence of large variations in both foreground and background across wide-baseline views a large amount of manual interaction is necessary for foreground segmentation. Video segmentation or matting approaches like $[1,2,3,9,10,13,14,15]$ can be used for foreground segmentation. These approaches are limited to single view video and require regular manual interaction every $10-30$ frames depending on the complexity of the given sequence. This means that the manual interaction required would increase by a factor equal to the number of cameras which would be prohibitively expensive for large sequences.

Video matting approaches like [1,3] used optical flow to propagate a manually defined coarse segmentation, referred to as a trimap, from the key-frames to the remaining frames in the sequence. For key-frames trimaps a coarse labelling of foreground and background pixels is manually defined. A matting approach is then used to refine this coarse segmentation to obtain a foreground silhouette. Motion estimation is not always accurate and could lead to erroneous segmentation requiring manual label correction at regular intervals $(10-20$ frames $)$ for a single view sequence. In [10] a template matching approach is used to exploit the local image features to propagate the segmentation without using motion estimates. This approach manages to reduce the user interaction but still requires regular input. A graph based approach that requires regular manually defined trimaps is proposed in [9]. The approach constructs a 3D graph at atomic level for the frames enclosed by the two key-frames. Global graph-cut optimisation is used to segment out the foreground object. This technique suffers in low contrast boundary region and proposed additional manual interaction in such cases. Another graph-cut based approach is proposed in [13]. This technique reduces the required manual interaction and improve the segmentation by incorporating contrast in the energy function. This approach produces unsatisfactory results if the foreground and background appearance become similar. A multi-view graph-cut based segmentation approach is proposed in [2]. The fundamental assumption of this technique is the fixation constraint: foreground should temporally occupy the same position in all the views. Satisfying this assumption is difficult and the technique also suffers in the low contrast boundary regions.

In this paper we propose a wide-baseline multi-view foreground segmentation algorithm using sparse user interaction on a single view captured by calibrated cameras. The user 


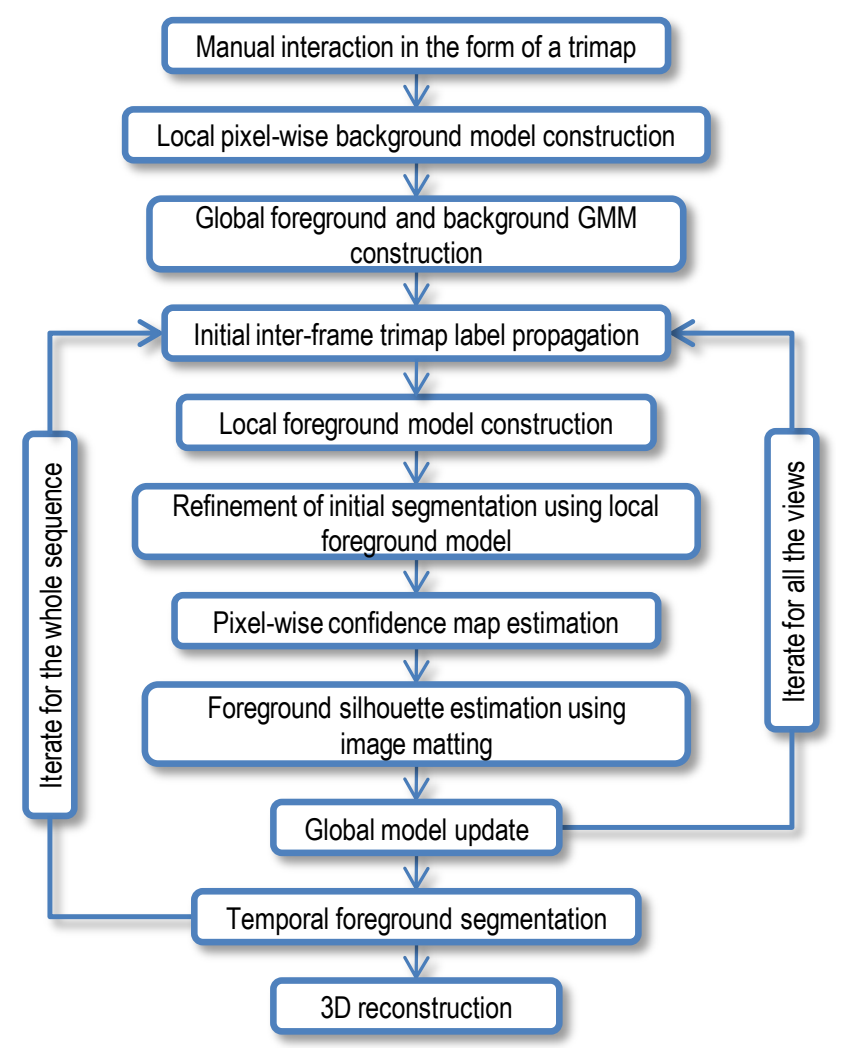

Figure 1: Flow diagram of the proposed algorithm

interaction is provided in the form of a coarse segmentation (trimap) defining foreground, background and ambiguous pixels in a key-frame. Trimap labels are spatio-temporally propagated and then refined by a matting algorithm to obtain foreground silhouettes across multiple views. The proposed algorithm significantly reduces the required user interaction to produce good results. Quantitative evaluation demonstrate that the proposed approach is capable of extracting a foreground silhouette for a view having a baseline upto $180^{\circ}$ from the key view (opposing views). We demonstrate how the proposed segmentation approach can be combined with the existing narrow baseline stereo reconstruction algorithms $[5,12]$ to extract $3 \mathrm{D}$ mesh sequences with interaction restricted to the definition of at most two trimaps for the full pipeline.

\section{WIDE-BASELINE SEGMENTATION}

Let us define the basic notations used in the paper. Frame at time $t$ in $N$ views $v$ are represented by $\left\{I^{t, v}\right\}_{v=1}^{N}$. The colour of a pixel $p$ in $I^{t, v}$ is represented $I_{p}^{t, v}$. The corresponding trimap of frames $\left\{I^{t, v}\right\}_{v=1}^{N}$ are represented by $\left\{\mathcal{T}^{t, v}\right\}_{v=1}^{N}$. The trimap label for a pixel $p$ in view $v$ is assigned as foreground $\mathcal{F}$, background $\mathcal{B}$ or ambiguous $\mathcal{U}$ and denoted by $\mathcal{T}_{p}^{t, v}$. A confidence map is also assigned to each trimap and its pixel-wise value is given as $\mathcal{C}_{p}^{t, v}$. A clean background plate for each view is denoted by $\left\{B^{v}\right\}_{v=1}^{N}$. This clean background can either be captured explicitly or can be learnt from the available video sequences. The proposed algorithm consists of the steps shown in the flow diagram 1. A detailed description of each step in the proposed wide-baseline segmentation algorithm is as follows.

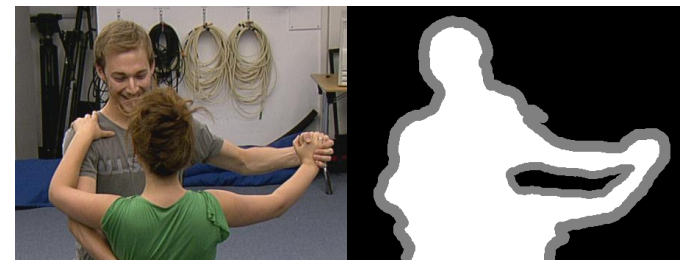

Figure 2: Trimap: A manual segmentation of a keyframe into foreground (white), background (black) and ambiguous (gray) regions.

\section{Step-1: Manual interaction.}

The user provides a definition of foreground, background and ambiguous pixels for a frame $I^{t, k}$ at time $t$ in a key view $k$. This coarse segmentation is known as a trimap and represented as $\mathcal{T}^{k}$. An example of a manually defined trimap can be seen in Fig 2. White and black represents the definite foreground and background pixels respectively. Due pixel spatial sampling resolution and overlap in foreground/background appearance in natural scenes trimap boundaries often contain pixels which can not be categorise either as foreground or background (gray pixels in Fig 2). These ambiguous pixels normally occur due to low camera resolution and motion blur caused by fast object movement or camera shake. These ambiguous pixels could lead to erroneous trimap propagation and hence are avoided by manually identifying them as ambiguous. A confidence map $\mathcal{C}^{t, k}$ is also associate to the trimap $\mathcal{T}^{k}$. All the definite foreground and background pixels are assigned the highest confidence of 1 while the ambiguous pixels are given a confidence of zero. Additional key-frame trimaps can be added to allow for temporal changes in foreground appearance.

\section{Step-2: Local pixel-wise background model $\mathcal{M}^{L B, v}$.}

Local background appearance is learnt for each view either a priori from a sequence of observations without foreground objects in the scene or in the case of a moving camera may be learnt online using motion estimation and per view image mosaicing [3]. Throughout this work we focus on the case of static cameras where the background appearance statistics for each pixel are learnt a priori from a short back ground sequence (20 frames). For each pixel we construct a GMM from the observed distribution to model the variation in background scene appearance due to movement, changes in illumination and camera noise throughout the sequence.

\section{Step-3: Global models $\left(\mathcal{M}^{G F}, \mathcal{M}^{G F}\right)$.}

Global appearance models generalise common appearance and variation in appearance between views reducing the requirement for key-frames on all the views. These global models lack information on appearance at a particular image location. As is common in the literature Gaussian Mixture Models (GMM) are used to represent the statistics of colour appearance for both the global and local models.

A global foreground Gaussian mixture model (GMM) is constructed for the pixels that are manually identified as foreground in the key-frame trimap $\mathcal{T}^{k}$. Any state-of-theart clustering algorithm can be used to construct $\mathcal{M}^{G F}$. We have used mean shift clustering technique [4] to obtain multivariate global foreground GMM in RGB colour space

A similar GMM is constructed to model the background appearance. A more precise pixel-wise local background model for each view is already constructed in step 2. Al- 

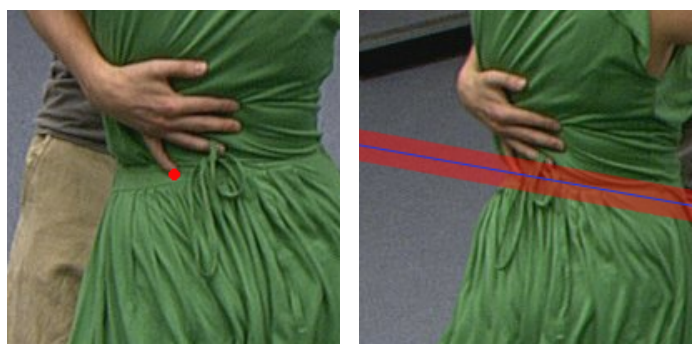

Figure 3: Epipolar band corresponding to a point in the first image is shown as a red band in the second image with epipolar line in the middle

though these local models are precise they can not model the variations observed due to the presence of foreground and temporal changes compared to the clean background plate. The global background GMM $\mathcal{M}^{G B}$ is used to model these variations. All the background pixels in the trimap $\mathcal{T}^{k}$ that are not modelled by the local background model $\mathcal{M}^{L B, k}$ are used to construct global background model $\mathcal{M}^{G B}$.

\section{Step-4: Initial inter-view trimap label propagation.}

Inter-view trimap label propagation at any given instance is formulated in Bayesian and inferential statistics framework. To increase the robustness in label propagation the epipolar constraint is also incorporated. Since camera calibration is a necessary requirement for $3 \mathrm{D}$ reconstruction the epipolar geometry is used to propagate the high confidence trimap labels from $\mathcal{T}^{k}$ to the neighbouring frame $I^{t, k+1}$.

Let us consider a pixel $q$ in the frame $I^{t, k+1}$, having a RGB colour value represented by $I_{q}^{t, k+1}$. If the calibration between the two cameras capturing the views $(k, k+1)$ is known then a pixel corresponding to $q$ in the other view at the same instance $I^{t, k}$ can be found on a line called epipolar line. This geometrical relation is known as epipolar constraint. To take into consideration possible calibration error the epipolar line is dilated by few pixels to form an epipolar band. Let us represent the epipolar band in $I^{t, k}$ corresponding to the pixel $q$ in $I^{t, k+1}$ by $\mathcal{R}_{q}^{t, k}$ and is shown in Fig 3.

As the foreground scene is a person or object who may move between successive frames it is not possible to model the local foreground statistics on a per-pixel basis a priori without knowledge of the foreground scene motion. If the global foreground and local background appearance at a particular pixel are similar a local foreground model of pixel statistics with a narrow per-pixel distribution is required to accurately label foreground pixels. The discussion on the local per pixels foreground model is delayed until step 5 .

It is critical to propagate trimap labels without the introduction of errors due to false positive foreground or background labels. In the case of pixels where there is an ambiguity between foreground and background model membership it is preferable to label them as unknown rather than incorrectly label pixels. It is referred as initial trimap propagation and results in a binary segmentation represented as $\mathcal{T}_{\text {init }}^{t, k+1}$. We have used statistical inference to test the pixel labelling hypothesis to within a strict confidence level (95\%). The foreground trimap label $\mathcal{F}$ is propagated to the pixels in the frame $I^{t, k+1}$ using maximum a posteriori, MAP, estimation of labels based appearance models. The posterior probability of the pixel $q$ belonging to the $i^{t h}$ component $\mathcal{M}_{i}\left(\mu_{i}, \Sigma_{i}\right)$ is given by Bayes theorem as:

$$
p\left(\mu_{i}, \Sigma_{i} \mid x=q\right)=\frac{p\left(x=q \mid \mu_{i}, \Sigma_{i}\right) p\left(\mu_{i}, \Sigma_{i}\right)}{p(x=q)}
$$

where $p\left(x=q \mid \mu_{i}, \Sigma_{i}\right)$ is the conditional probability of pixel $q$ given the model component $\mathcal{M}_{i}$ with mean $\mu_{i}$ and covariance $\Sigma_{i}$. The term $p\left(\mu_{i}, \Sigma_{i}\right)$ is the prior for the $i^{t h}$ cluster. The constant $p(x=q)$ is the prior for pixel $q$.

To find the most likely cluster, $\mathcal{M}_{m l}$ with MAP estimates $\left(\mu_{m l}, \Sigma_{m l}\right),(1)$ is maximised over a partial set of global model components $\mathcal{M}_{j}$. This partial set only contain the components that belong to the epipolar band $\mathcal{R}_{q}^{t, k}$, for pixel $q$, in the frame $I^{t, k}$. Maximisation of (1) is performed as

$$
\left(\mu_{m l}, \Sigma_{m l}\right)_{\mathcal{M}_{m l}}=\underset{\mathcal{M}_{j}}{\arg \max } p\left(x=q \mid \mu_{j}, \Sigma_{j}\right) p\left(\mu_{j}, \Sigma_{j}\right) .
$$

The denominator in (1) is independent of the cluster parameters and therefore removed from the optimisation. Separate MAP estimates are made using equation (2) over the global foreground $\mathcal{M}^{G F}$, global background $\mathcal{M}^{G B}$ models for pixel $q$ in view $k+1$ to find the most likely global foreground and background components, $\mathcal{M}_{m l}^{G F}, \mathcal{M}_{m l}^{G B}$ respectively. For the sake of consistency we refer the local background model for pixel $q$ by $\mathcal{M}_{m l}^{L B}$.

In a multivariate GMM the MAP estimate corresponds to the minimum squared Mahalanobis distances $\mathcal{Q}_{\text {min }}$. The squared Mahalanobis distance, $\mathcal{Q}$, follows the chi-square distribution over $d$ degrees of freedom that is $\mathcal{Q} \sim \chi^{2}(d)$, where $d=3$ is the dimensionality of the colour space. We use statistical inference based on the $\chi^{2}$ test to infer the trimap label with a $95 \%$ confidence level for a pixel $q$ as foreground $\mathcal{F}$ otherwise the pixel is labelled as ambiguous $\mathcal{U}$. We define three separate null hypothesis tests for a pixel $q$ to belong to, either $\mathcal{M}_{m l}^{G F}, \mathcal{M}_{m l}^{G B}$ or $\mathcal{M}_{m l}^{L B}$ as:

$$
\begin{array}{lll|l}
\mathcal{H}_{0}^{G F} & : & q \in \mathcal{M}_{m b}^{G F} & \mathcal{Q}_{m i n}^{G F} \leq \chi_{\gamma, d}^{2} \\
\mathcal{H}_{0}^{G B} & : & q \in \mathcal{M}_{m b}^{G B} & \mathcal{Q}_{m i n}^{G B} \leq \chi_{\gamma, d}^{2} \\
\mathcal{H}_{0}^{L B} & : & q \in \mathcal{M}_{m l}^{L B} & \mathcal{Q}_{m i n}^{L B} \leq \chi_{\gamma, d}^{2}
\end{array}
$$

where $\chi_{\gamma, d}^{2}$ is the critical value for the $\chi^{2}$ distribution over $d$ degrees of freedom at the significance level of $\gamma=0.05$.

The trimap label is propagated to the pixel $q$ in the trimap $\mathcal{T}_{\text {init }}^{t, k+1}$ as

$$
\mathcal{T}_{\text {init }}^{t, k+1}(q)= \begin{cases}\mathcal{F} & \text { if, }\left(\mathcal{H}_{0}^{G F}\right) \wedge\left(\neg \mathcal{H}_{0}^{G B}\right) \wedge\left(\neg \mathcal{H}_{0}^{L B}\right) \\ \mathcal{U} & \text { otherwise. }\end{cases}
$$

$\mathcal{T}_{\text {init }}^{t, k+1}(q)$ is the initial trimap label for pixel $q$ in view $k+$ 1 at time $t$. Initial trimap label propagation is performed independently over all the pixels in $I^{t, k+1}$.

The use of a statistical hypothesis test with a given confidence level ensures that only high-confidence labels are propagated and false-positive trimap pixel labels in the resulting trimap are extremely rare. An example initial trimap propagation is presented in Fig 4(b).

\section{Step-5: Local foreground model construction.}

Due to the absence of a local foreground model in the initial trimap propagation there will be holes in the foreground where it is similar to the local or global background model Fig 4(b). A significant improvement in trimap labelling can be obtained by modelling the local foreground appearance. One possibility as used in previous single-view video matting approaches $[1,3]$ is to estimate the inter-frame motion using 

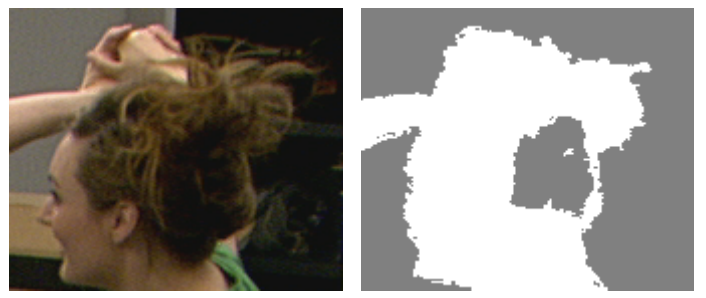

(a) Image region

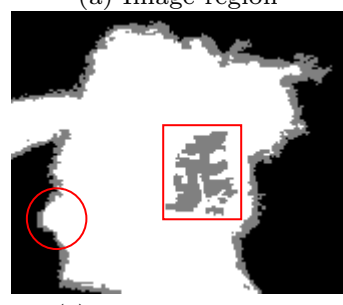

(c) Final trimap est. (b) Initial trimap est.

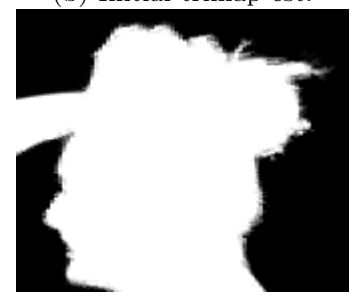

(d) Silhouette using [8]

Figure 4: Example of trimap estimation.

optic flow for temporal propagation of trimap information. However, motion estimation for an arbitrarily moving person in a natural scene is a complex problem and standard optic flow techniques produce low-quality frame-to-frame transfer. This has resulted in the need for extensive manual interaction in previous single view trimap propagation with manually labelled key-frames required every $10-20$ frames. In the case of multiple view trimap propagation this would prove prohibitively expensive.

We have used the initial trimap foreground labels to estimate a local per-pixel foreground model $\mathcal{M}^{L F, t, k+1}$. To construct a local foreground model for an ambiguous pixel $q$, we localise a circular window $\omega(q)$ at pixel $q$. All the definite foreground pixels in $\omega(q)$ are modelled as Gaussian mixture to form local foreground model for pixel $q$. This local model is then used to fill the foreground holes and perform refinement of initial trimap propagation.

\section{Step-6: Refinement using local foreground model.}

After obtaining the local foreground model refinement of initial trimap can be performed to reassign labels to the unknown pixels in (4). To reassign a trimap label to an unknown pixel $q$ we define an additional null hypothesis test for the local foreground:

$$
\mathcal{H}_{0}^{L F} \quad: \quad q \in \mathcal{M}_{m l}^{L F} \quad \mid \quad \mathcal{Q}_{\text {min }}^{L F} \leq \chi_{\gamma, d}^{2}
$$

The final trimap label assignment is then performed using the complete set of hypothesis tests for local and global models:

$$
\mathcal{T}^{t, k+1}(q)= \begin{cases}\mathcal{F} & \text { if, }\left(\mathcal{H}_{0}^{G F} \vee \mathcal{H}_{0}^{L F}\right) \wedge\left(\neg \mathcal{H}_{0}^{G B}\right) \wedge\left(\neg \mathcal{H}_{0}^{L B}\right) \\ \mathcal{B} & \text { if, }\left(\mathcal{H}_{0}^{G B} \vee \mathcal{H}_{0}^{L B}\right) \wedge\left(\neg \mathcal{H}_{0}^{G F}\right) \wedge\left(\neg \mathcal{H}_{0}^{L F}\right) \\ \mathcal{U} & \text { otherwise. }\end{cases}
$$

Fig 4(c) shows the refined trimap where the foreground pixels previously labelled as ambiguous (Fig 4(b)) are correctly labelled shown by red markers in Fig 4(c). This shows that the estimated trimap gives a good approximation of the foreground object boundary.

\section{Step-7: Confidence map estimation.}

Estimation of per-pixel trimap label confidence is critical

to the propagation between views and over time using statistical inference. This confidence is used to dynamically update the global appearance models incorporating new observations based on the label confidence to avoid drift. We associate a confidence level to each pixel $q$ in the trimap $\mathcal{T}^{t, k+1}$ to construct the confidence map $\mathcal{C}^{t, k+1}$. Let us consider that pixel $q$ has foreground label. The confidence is formulated using the maximum likelihood foreground and background model component given by equation (2) over both local and global models. The confidence for pixel $q$ is estimated as:

$$
\mathcal{C}^{t, k+1}(q)=\frac{e^{-\mathcal{Q}_{m i n}^{F} / 2}}{(2 \pi)^{3 / 2}\left|\Sigma_{m l}^{F}\right|^{1 / 2}}\left(1-\frac{e^{-\mathcal{Q}_{m i n}^{B} / 2}}{(2 \pi)^{3 / 2}\left|\Sigma_{m l}^{B}\right|^{1 / 2}}\right) p\left(\mu_{m l}^{F}, \Sigma_{m l}^{F}\right) .
$$

The introduction of the prior confidence level $p\left(\mu_{m l}^{F}, \Sigma_{m l}^{F}\right)$ ensures that the confidence of pixel labels used to dynamically update clusters is taken into account so that the model does not drift. The confidence for a background pixel is estimated in the same way by interchanging the foreground and background terms in (7).

\section{Step-8: Foreground silhouette estimation.}

It is an optional step to further refine the foreground boundary, to obtain the final foreground silhouette $\mathcal{S}^{t, k+1}$, by applying any image matting algorithm. In this paper we have used Levin et al.'s closed form solution [8] to obtain an alpha matte $\alpha^{t, k+1}$ of $I^{t, k+1}$ using the propagated trimap $\mathcal{T}^{t, k+1}$. An alpha matte represents the foreground opacity ranging $[0,1]$. The definite foreground and background pixels have an alpha value of 1 and 0 respectively while fractional alpha values represents the mixed foreground and background pixels. The foreground silhouette $\mathcal{S}^{t, k+1}$ is obtained by applying a predefined threshold $\epsilon^{\alpha}$ as

$$
\mathcal{S}^{t, k+1}(i)= \begin{cases}1 & \mid \alpha^{t, k+1}(i)>\epsilon^{\alpha} \\ 0 & \text { otherwise }\end{cases}
$$

The foreground silhouette $\mathcal{S}^{t, k+1}$ is a binary mask representing foreground pixels by 1 as shown in Fig 4(d).

\section{Step-9: Global model update.}

The estimated confidence of trimap label is used to update the global foreground and background models. This allows to incorporate novel appearance information and to limit drift over time. In practice this is required to allow the model to incorporate differences in foreground and background appearance due to viewpoint and changes due to shadows and shading over time. Model update is limited to foreground and background pixels which are not represented with high-confidence in the prior global models. The new clusters obtained are appended to the prior models. This updating approach allows the incorporation of novel appearance information without reducing the confidence of models derived from the manually specified key-frame trimap.

After foreground segmentation is performed on all the views at time $t=t$, there could be a large number of components of low confidence in the global Gaussian mixture models. These low confidence clusters could produce misclassification errors in temporal trimap label propagation to the frame $I^{t+1, k}$. We enforce a strict criteria, to limit error propagation, that a low confidence cluster must be observed in atleast $l=3$ views at time $t$. The Bhattacharyya coefficient for multivariate Gaussian distribution is used to 
Root mean square error in different views for different sequences

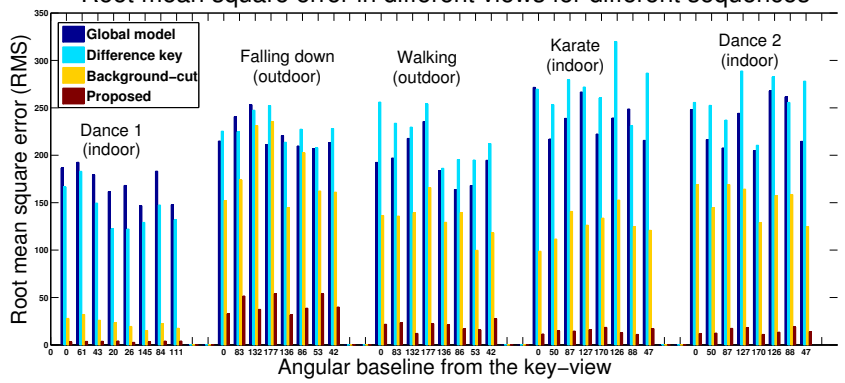

Figure 5: Root mean square error, RMS, for different sequences.

find the consistency of a low confidence cluster. The Bhattacharyya coefficient is a function of mean and covariance of the two Gaussian clusters which measures their overlap. Only the clusters that fulfil the consistency criteria are combined together and introduced as a new component in the respective global model prior to the temporal propagation.

\section{Step-10: Temporal Foreground Segmentation.}

The epipolar constraint used in optimisation in (2) enforce consistency of colour labels between views at each time step. For temporal propagation of trimap labels, from $I^{t, k}$ to $I^{t+1, k}$, we apply optimisation in (2) on the entire set of components in global foreground and background models. The optimisation is performed for each pixel in the frame $I^{t+1, k}$.

\section{Step-11: 3D Reconstruction.}

Given the segmentation across all views at each time frame the scene can be reconstructed using visual hull and refined using correspondence between views. The visual hull is obtained by backprojecting the segmented foreground regions in $3 \mathrm{D}$ space and computing the intersection of the cone they defined. This approach is known to produce an approximate scene representation which is guaranteed to contain the true scene surface. A main limitation of the visual hull however is its inability to reconstruct concavities and the existence of phantom volumes (see Fig 6). As such, visual hull reconstruction must be followed by dense stereo refinement using correspondence between views.

The main idea behind stereo recontruction is to use textural information to establish correspondences between images and thereby obtain a more accurate scene reconstruction (see Fig 6). In this work reconstruction is performed using techniques previously developed for wide-baseline 3D reconstruction $[5,12]$. Both approaches use graph-cut optimisation techniques in order to compute an optimum surface reconstruction, using the visual hull as an initial estimate. In [12] the problem is formulated as a global volumetric optimisation problem where all cameras are simultaneously considered, while [5] performs a view-dependent optimisation from each camera view-point and then merges the outputs into a unique representation using Poisson surface reconstruction. Both approaches result in a $3 \mathrm{D}$ video mesh sequence reconstruction at each time-frame.

\section{RESULTS AND EVALUATION}

Results are presented for wide-baseline foreground segmentation for challenging natural indoor and outdoor scenes.
The sequences contain one or more moving people performing rapid movement and have complex elements such as hair and loose clothing. A circular rig of 8 cameras is used with an angular separation of around $45^{\circ}$. The first row of Fig 6 shows the full high definition key views used for the different sequences. We have used $2 \mathrm{key}$-frames in a single view for the indoor sequences consists of 250 frames per view. For the outdoor sequences having length of 125 frames per view only one key-frame in a single view is used. A comparative evaluation is performed between four different approaches: (1) global model comparison using foreground and background models only from the key frame, (2) difference keying, (3) background cut [13], and (4) proposed segmentation algorithm. The ground truths are estimated for frames at every second in each view using a precise hand drawn trimap and using [10]. The obtained foreground segmentation is shown in Fig 6 with error from ground-truth in red.

The segmentation performed by difference keying is severely contaminated by shadows and uncontrolled outdoor environment. The global model approach has reduced the artifact especially in the outdoor scene but still suffers where there is an overlap between the global foreground and background models (actor's shirt and carpet). The parameters of background cut [13] are tuned to get the best results but still it is unable to classify the shadow region as background and suffers where the foreground model is similar to the local pixelwise background model. Our technique is able to reduce the errors caused by the overlap in the models by incorporating the local foreground model. The results obtained show few visible artifacts compared to the ground truth.

To compare the techniques quantitatively we have used root mean square error, RMS. The ground truth segmentation is obtained for every second of video for all the views. The error measure is shown in Fig. 5. It is clear from the comparison that the proposed segmentation algorithm outperform other techniques.

Reconstruction results obtained using the techniques described in step 11 are shown in Fig 6. To demonstrate the modularity of the proposed pipeline, reconstruction has been performed using two different reconstruction algorithms: view dependent graph-cuts [5] (used for dance1 and dance2 sequences) and volumetric graph-cut [12] (used for the remaining sequences). In each case, the stereo reconstruction algorithm was initialised using the visual hull computed from the estimated segmentation as described in Step 2. The results obtained demonstrate the suitability of the technique for multi-view reconstruction with wide-baseline camera setups and natural indoor and outdoor scenes. In particular, the technique significantly reduces the need for user interaction normally needed during segmentation. In all the results presented, only a maximum of two manual trimaps in a single view are used and no further manual intervention is required for segmentation and reconstruction.

\section{CONCLUSION}

A novel wide-baseline spatio-temporal foreground segmentation algorithm has been introduced based on a Bayesian inference framework. Key-frame trimaps are specified in a single view and robustly propagated across wide-baseline views using label confidence to perform segmentation. Results are presented which demonstrate high-quality segmentation for video sequences with views up to $180^{\circ}$ from the key view. The technique only requires manual trimap in- 


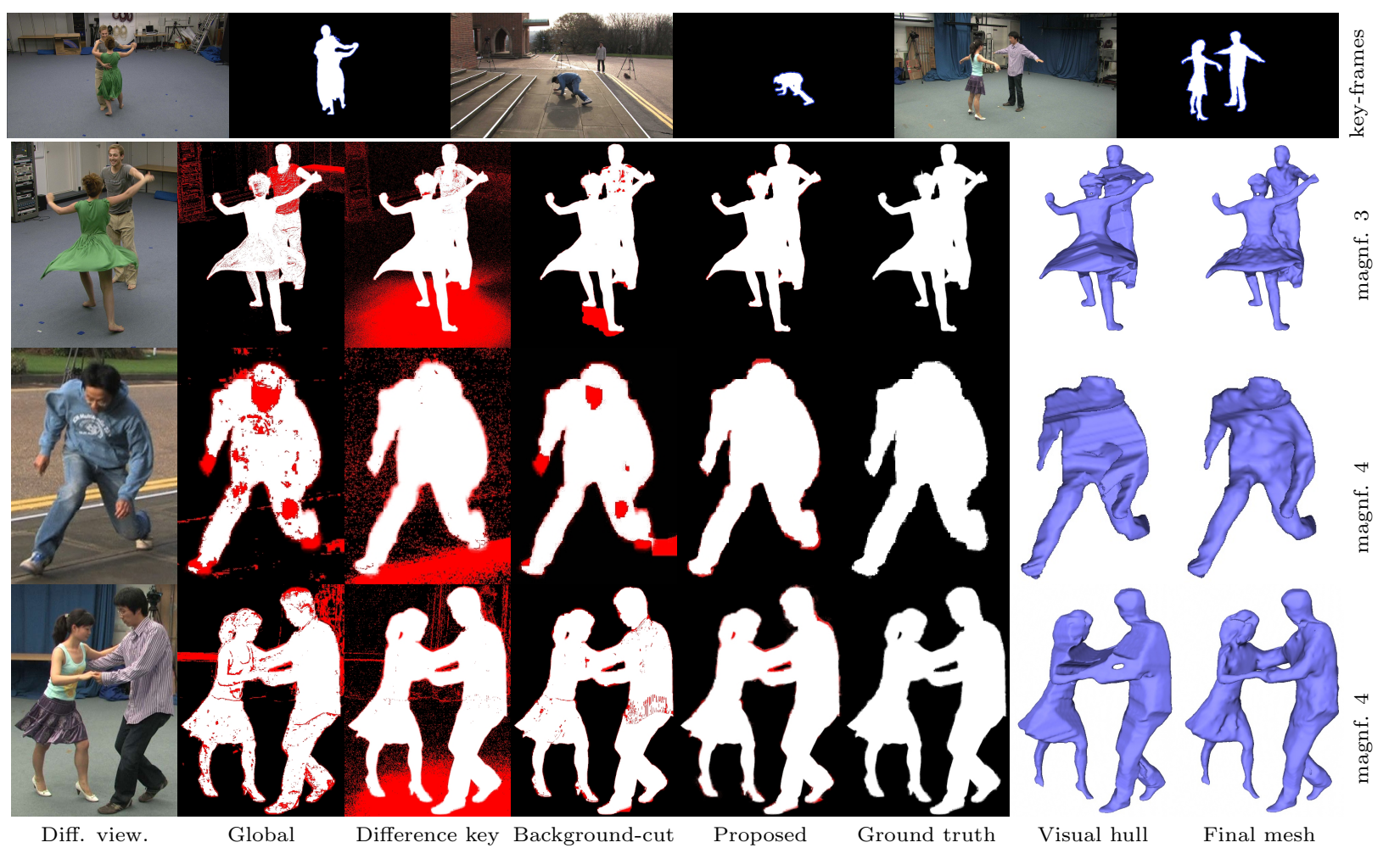

Figure 6: Segmentation obtained using different approaches along with the ground truth and 3D mesh using proposed segmentation. First row shows the different key-frames used along with the hand drawn trimaps. The segmentation error is shown in red.

put for 1-2 key-frames in a single view to process sequences of several hundred frames over 8 views. This is a significant reduction in manual interaction compared with stateof-the-art video segmentation. Segmentation is sufficiently accurate to allow 3D reconstruction of both indoor and outdoor scenes with complex natural backgrounds. The proposed algorithm can be integrated seamlessly into any 3D reconstruction system.

\section{REFERENCES}

[1] N. E. Apostoloff and A. W. Fitzgibbon. Bayesian video matting using learnt image priors. In $C V P R$, pages 407-414, 2004.

[2] N. D. F. Campbell, G. Vogiatzis, C. Hernandez, and R. Cipolla. Automatic 3d object segmentation in multiple views using volumetric graph-cuts. Image and Vision Computing, 2008.

[3] Y. Y. Chuang, A. Agarwala, B. Curless, D. Salesin, and R. Szeliski. Video matting of complex scenes. In Proceedings of ACM SIGGRAPH, pages 243-248, 2002.

[4] D. Comaniciu and P. Meer. Mean shift: a robust approach toward feature space analysis. Pattern Analysis and Machine Intelligence PAMI, 24(5):603-619, 2002.

[5] J.-Y. Guillemaut, J. Kilner, and A. Hilton. Robust graph-cut scene segmentation and reconstruction for free-viewpoint video of complex dynamic scenes. In $I C C V, 2009$.

[6] M. Hyun, S. Kim, and Y. Ho. Multi-view image matting and compositing using trimap sharing for natural 3-d scene generation. In $3 D T V$, pages 397-400, 2008.

[7] N. Joshi, W. Matusik, and S. Avidan. Natural video matting using camera arrays. ACM Transaction on Graphics, 25(3):779-786, 2006.

[8] A. Levin, D. Lischinski, and Y. Weiss. A closed form solution to natural image matting. CVPR, 1:61-68, 2006.

[9] Y. Li, J. Sun, and H. Y. Shum. Video object cut and paste. ACM Trans. Graph., 24(3):595-600, 2005.

[10] M. Sarim, A. Hilton, and J.-Y. Guillemaut. Non-parametric patch based video matting. In $B M V C$, 2009.

[11] P. Narayanan, P. Rander, and T. Kanade. Constructing virtual worlds using dense stereo. In ICCV, pages 3-10, 1998.

[12] J. Starck and A. Hilton. Surface capture for performance-based animation. IEEE Computer Graphics and Applications, 27(3):21-31, 2007.

[13] J. Sun, W. Zhang, X. Tang, and H.-Y. Shum. Background cut. In $E C C V$, pages $628-641,2006$.

[14] J. Wang, P. Bhat, R. A. Colburn, M. Agrawala, and M. F. Cohen. Interactive video cutout. ACM Trans. Graph., 24(3):585-594, 2005.

[15] L. C. Zitnick, S. B. Kang, M. Uyttendaele, S. Winder, and R. Szeliski. High-quality video view interpolation using a layered representation. ACM Trans. Graph., 23(3):600-608, 2004. 